\title{
SYNTHETIC LINE PROFILES FROM UNIFIED MODEL ATMOSPHERES
}

\author{
E. SANTOLAYA REY and A. HERRERO \\ Instituto de Astrofísica de Canarias, E-38200 La Laguna, Tenerife, Spain \\ and \\ J. PULS \\ Institut für Astronomie und Astrophysik, Scheinerstraße 1, D-81679 München, B.R.D.
}

\begin{abstract}
Synthetic line-profiles from unified model atmospheres are presented. The main characteristics of the code are also shown concerning both the calculation of NLTE populations (in a consistent way with the radiation field), and the formal solution to obtain emergent profiles. Stark-broadening is considered in this last step. Emergent $\mathrm{H} \alpha$ profiles for a grid of models are shown for $T_{\text {eff }}=50000 \mathrm{~K}$, covering a wide range of stellar radii and mass-loss.
\end{abstract}

Key words: stars: atmospheres - early type - mass-loss - radiative transfer - line-profiles

\section{The model}

Wolf-Rayet stars show evidence for dense, supersonic winds. We have developed a unified model (i.e., a model in which there is no separation between photosphere and wind) for solving the radiation transfer in this atmospheres. We assume spherical geometry and an analytical velocity law, parametrized according to Hamann \& Schmutz (1987). We use a grey LTE temperature with a cut-off at a certain minimun temperature. The atomic models we are considering include all the ionization states of $\mathrm{H}$ and $\mathrm{He}$, with a total number of 53 NLTE levels.

For continuum transfer a spherical statical solution is calculated. The rate equations are solved in the framework of the ALI-iteration with our own preconditioning. Line-transfer is solved under the Sobolev plus continuum approach (Puls \& Hummer 1987) and the actual continuum intensities enter on the calculation of the scattering integral.

After calculating the occupation numbers consistently with the temperature and the radiation field, a formal solution is performed, in the $p-z$ geometry, to obtain emergent profiles. It is based in the concept of resonant zones as developed in the Sobolev theory, allowing for doublets and overlapping lines. In order to maintain spectral resolution, a micro-grid is calculated over every ray. Depth-dependent Stark profiles (Schöning \& Butler 1989) are considered, both in the location of resonant zones and in integration over the ray. 


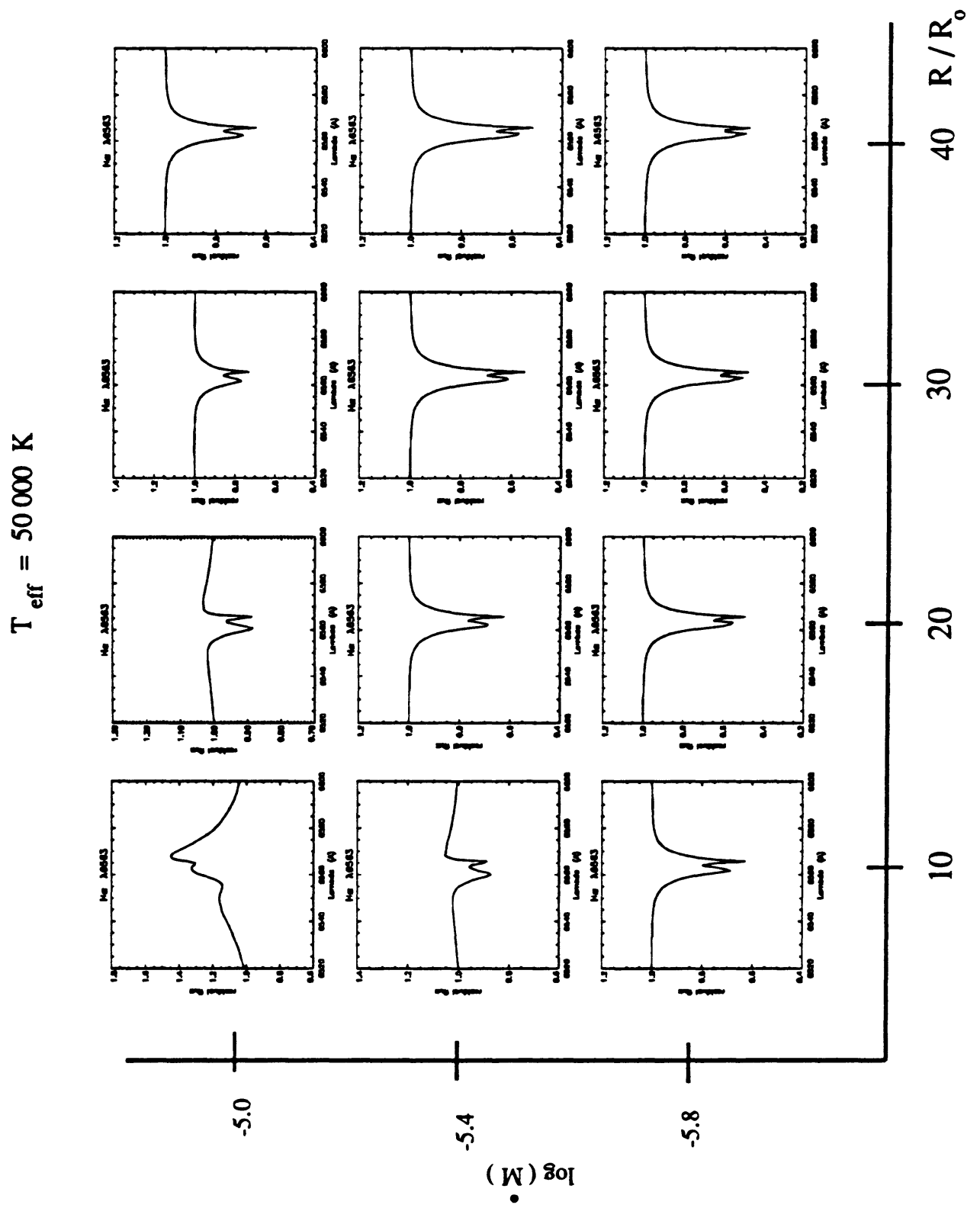

Fig. 1. Synthetic $\mathrm{H} \alpha$ profiles for models with $T_{\text {eff }}=50000 \mathrm{~K}$, radii between 10 and $40 \mathrm{R}_{\odot}$, and mass-loss between $10^{-6.6}$ and $10^{-5} \mathrm{M}_{\odot} \mathrm{yr}^{-1}$

\section{References}

Puls, J., Hummer, D. 1987, $A \& A$ 191, 87

Hamann, W.R., Schmutz, W. 1987, $A \& A$ 174, 173

Schöning, T., Butler, K. 1989, $A \mathscr{B} A$ 219, 362 